\title{
Radiation Recall Reaction (Dermatologic), CTCAE
}

National Cancer Institute

\section{Source}

National Cancer Institute. Radiation Recall Reaction (Dermatologic), CT CAE. NCI

Thesaurus. Code C143796.

A finding of acute skin inflammatory reaction caused by drugs, especially chemotherapeutic agents, for weeks or months following radiotherapy. The inflammatory reaction is confined to the previously irradiated skin and the symptoms disappear after the removal of the pharmaceutical agent. 